\title{
Chiral extrapolation of elastic meson-meson scattering: phase shifts and resonance poles
}

\author{
J. Nebreda and J.R. Peláez \\ Dept. Física Teórica II. Universidad Complutense, 28040, Madrid. Spain
}

\begin{abstract}
We study the dependence of the $\pi \pi$ scattering phase shifts on the light quark mass in both standard and unitarized SU(2) Chiral Perturbation Theory (ChPT) to one and two loops. We then use unitarized SU(3) ChPT to study the elastic $f_{0}(600), \kappa(800), \rho(770)$ and $K^{*}(892)$ resonances. The quark masses are varied up to values of interest for lattice studies. We find a very soft dependence on the light quark mass of the $\pi \pi$ phase shifts as a function of the center of mass momentum at one loop and slightly stronger at two loops and a good agreement with lattice results. The SU(3) analysis shows that the properties of the $\rho(770)$ and $K^{*}(892)$ depend smoothly on the quark mass whereas the scalar resonances present a non-analyticity at high quark masses. We also confirm the lattice assumption of quark mass independence of the vector two-meson coupling that, however, is violated for scalars.
\end{abstract}

PACS: 14 .40.Cs, $12.39 . \mathrm{Fe}, 13.75 . \mathrm{Lb}$

Standard ChPT. We use the SU(2) scattering amplitudes in [1] and the LECs in [4] to study the dependence of the $\pi \pi$ phase shifts on the averaged $u$ and $d$ quark mass, $\hat{m}$. In Fig. 1 we show preliminary results for phases on different channels for different pion masses, to one and two loops. They are plotted as a function of the center of mass momentum, and not of the energy, in order to subtract the shift of the thresholds. Since the applicability of plain ChPT is limited to low energies, we do not plot phase shifts beyond $\sqrt{s}=1 \mathrm{GeV}$. Note the agreement with the few existing lattice results for the $\mathrm{I}=2$, $\mathrm{J}=0$ channel [5], although for that comparison we use $M_{\pi}=420 \mathrm{MeV}$, which is most likely beyond the applicability region. A Montecarlo gaussian sampling based on the errors of the LECs has been used to calculate the error bands. The dependence of the phase shifts on $\hat{m}$ is very soft at one loop and somehow stronger at two loops.

Unitarized ChPT. We use now the IAM to unitarize our amplitudes. This procedure extends their applicability up to the resonance region and generates poles in the second Riemann sheet, which relate to resonances through the usual Breit-Wigner identification $\sqrt{s_{\text {pole }}} \equiv$ $M-i \Gamma / 2$. Furthermore, the coupling of the resonance to two mesons is given by the residue of the amplitude at the pole position.

Phase shifts. In Fig.2 we show preliminary results for $\pi \pi$ phase shifts (note that the one- and two-loop IAM cannot be used for D-waves) for different pion masses. For the one-loop analysis we used the LECs in [2] and found once again that the dependence is quite soft, specially for the $\mathrm{I}=2, \mathrm{~J}=0$ channel. For the two-loop analysis we used the LECs from fit D in [6]. Again the dependence on $M_{\pi}$ is somewhat stronger at two loops.
Resonance dependence on the quark masses in $S U(3)$ [3]. In Fig.3 we show the dependence of the $\rho(770), K^{*}(892), f_{0}(600)$ and $\kappa(800)$ on $\hat{m}$. The masses of the vector resonances increase smoothly, but much slower than $M_{\pi}$. As a consequence there is a strong phase space suppression which accounts by itself for the width decrease, without a dynamical effect through the couplings $g_{\rho \pi \pi}$ and $g_{K^{*} \pi K}$, that are remarkably constant, which is an assumption made in lattice studies of the $\rho(770)$ width [8]. On the other hand, the $f_{0}(600)$ and the $\kappa(800)$ scalar mesons behave quite differently. Their mass split into two branches when the associated pair of conjugated poles in the second Riemann sheet, which are approaching each other as $\hat{m}$ increases, join in a single pole below threshold to split again and remain in the real axis. Quantitatively, the growth of the $f_{0}(600)$ mass before the "splitting point" is much faster than that of the $\kappa(800)$. Their width decrease cannot be attributed to the phase space reduction now, because their coupling to two mesons depend strongly on $\hat{m}$. Finally, let us remark that the results obtained in the $\pi \pi$ channel are very consistent with those in SU(2) [2] and the estimations for the two first coefficients of the $M_{\rho}$ chiral expansion [7].

Resonance dependence on the strange quark mass [3]. In Fig. 3 we show the dependence of the same resonances on the strange quark mass $m_{s}$ in the limited range $0.8<$ $m_{s} / m_{s \text { phys }}<1.4$ to ensure that the kaon does not become too heavy to spoil the ChPT convergence nor too light to require a coupled channel formalism. As expected, the properties of the $\rho(770)$ and sigma non-strange resonances are almost independent of $m_{S}$ within the range of study. Obviously, the $K^{*}(892)$ and the $\kappa(800)$ show 

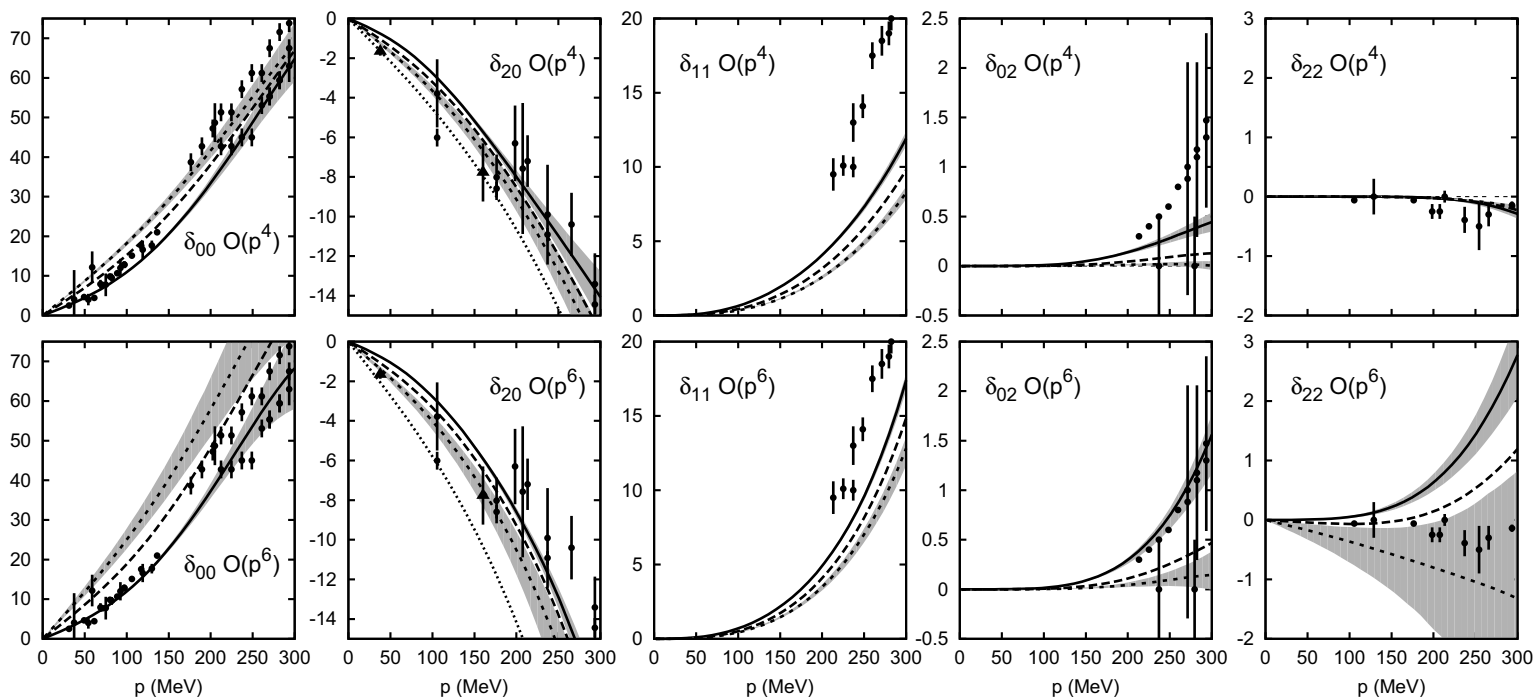

FIGURE 1. $\pi \pi$ phase shifts from standard ChPT up to one loop (first row) and to two loops (second row). Continuous, long dashed, short dashed and dotted (only in $\delta_{20}$ ) stand for $M_{\pi}=139.57,230,300$ and $420 \mathrm{MeV}$. We do not plot phase shifts beyond $\sqrt{s}=1 \mathrm{GeV}$. References for experimental data (circles) can be found in [3]. Lattice results for $\delta_{20}$ (triangles) come from [5].

a strong dependence. As the kaon mass is made heavier, their masses grow much faster than they did when increasing $\hat{m}$, but still much slower than the kaon mass. As before, in the case of the vector resonance the width decreases almost exactly as it would be expected from phase space suppression only and its coupling to $K \pi$ is almost constant, while the scalar width decrease deviates significantly from that behavior, in agreement with $g_{\kappa} \pi K$ depending quite strongly on $m_{s}$.

Acknowledgments. Work supported by Spanish MICINN FPA2008-00592 and FIS2006-03438, UCMBSCH GR58/08 910309 and the HadronPhysics2 grant n 227431 of the EU Seventh Framework Programme.

\section{REFERENCES}

1. J. Bijnens, G. Colangelo, G. Ecker, J. Gasser and M. E. Sainio, Phys. Lett. B 374, 210 (1996).

2. C. Hanhart, J. R. Pelaez and G. Rios, Phys. Rev. Lett. 100, 152001 (2008).

3. J. Nebreda and J. R. Pelaez., Phys. Rev. D 81, 054035 (2010).

4. G. Colangelo, J. Gasser and H. Leutwyler, Nucl. Phys. B 603, 125 (2001).

5. K. Sasaki and N. Ishizuka, Phys. Rev. D 78, 014511 (2008)

6. J. R. Pelaez, G. Rios, [arXiv:1010.6008 [hep-ph]], to appear in Phys. Rev. D.

7. P. C. Bruns and U.-G. Meißner, Eur. Phys. J. C 40 (2005) 97.

8. S. Aoki et al. [CP-PACS Collaboration], Phys. Rev. D 76, 094506 (2007).
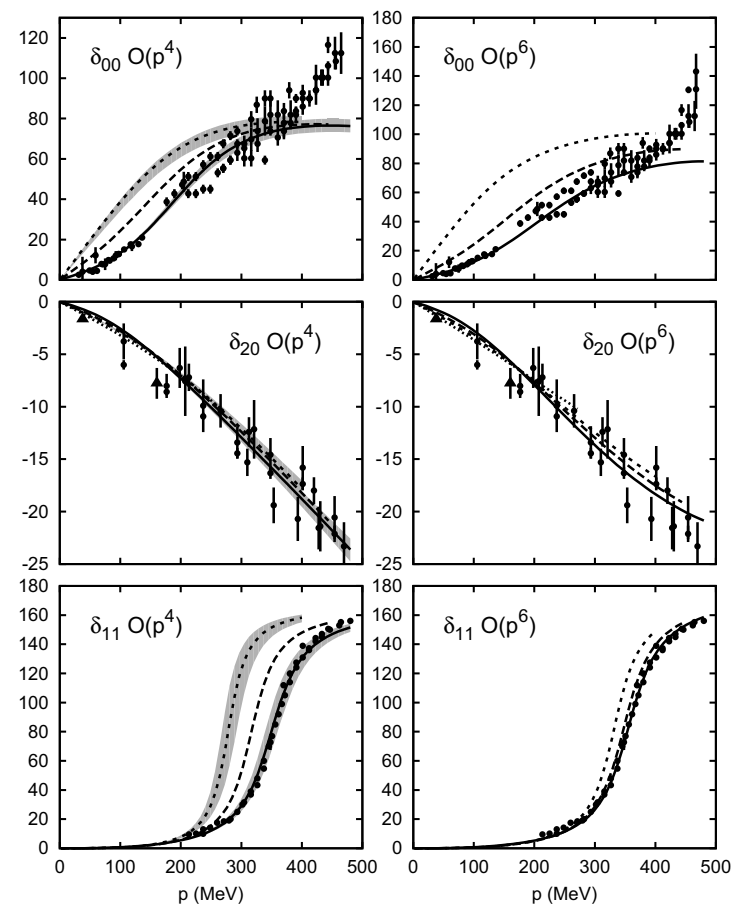

FIGURE 2. $\pi \pi$ phase shifts from unitarized ChPT up to one loop (first row) and to two loops (second row). The conventions are as in Fig. 2. For the one-loop analysis we use the LECs in [2] and for the two-loop one we use the LECs from fit D in [6]. 

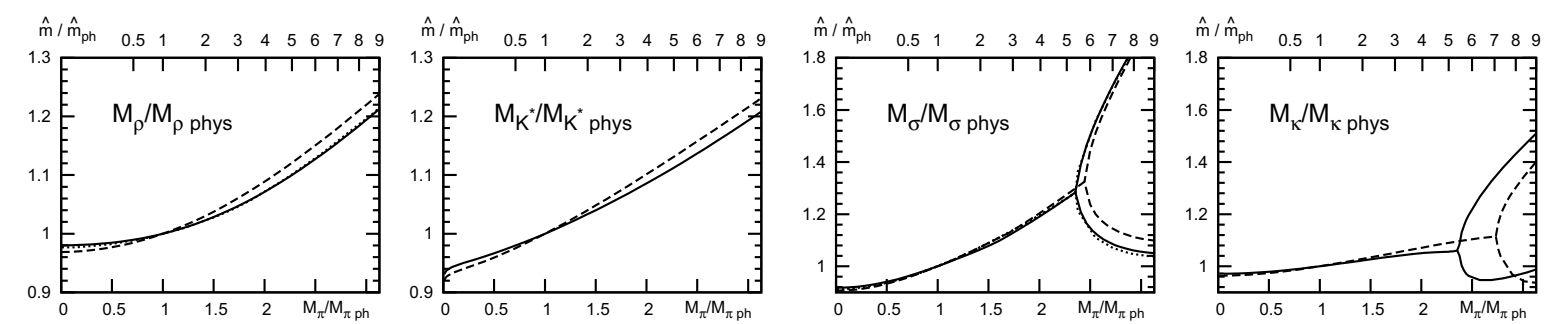

$\hat{\mathrm{m}} / \hat{\mathrm{m}}_{\mathrm{ph}} \quad \begin{array}{lllllllllll} & 0.5 & 1 & 2 & 3 & 4 & 5 & 6 & 7 & 8 & 9\end{array}$
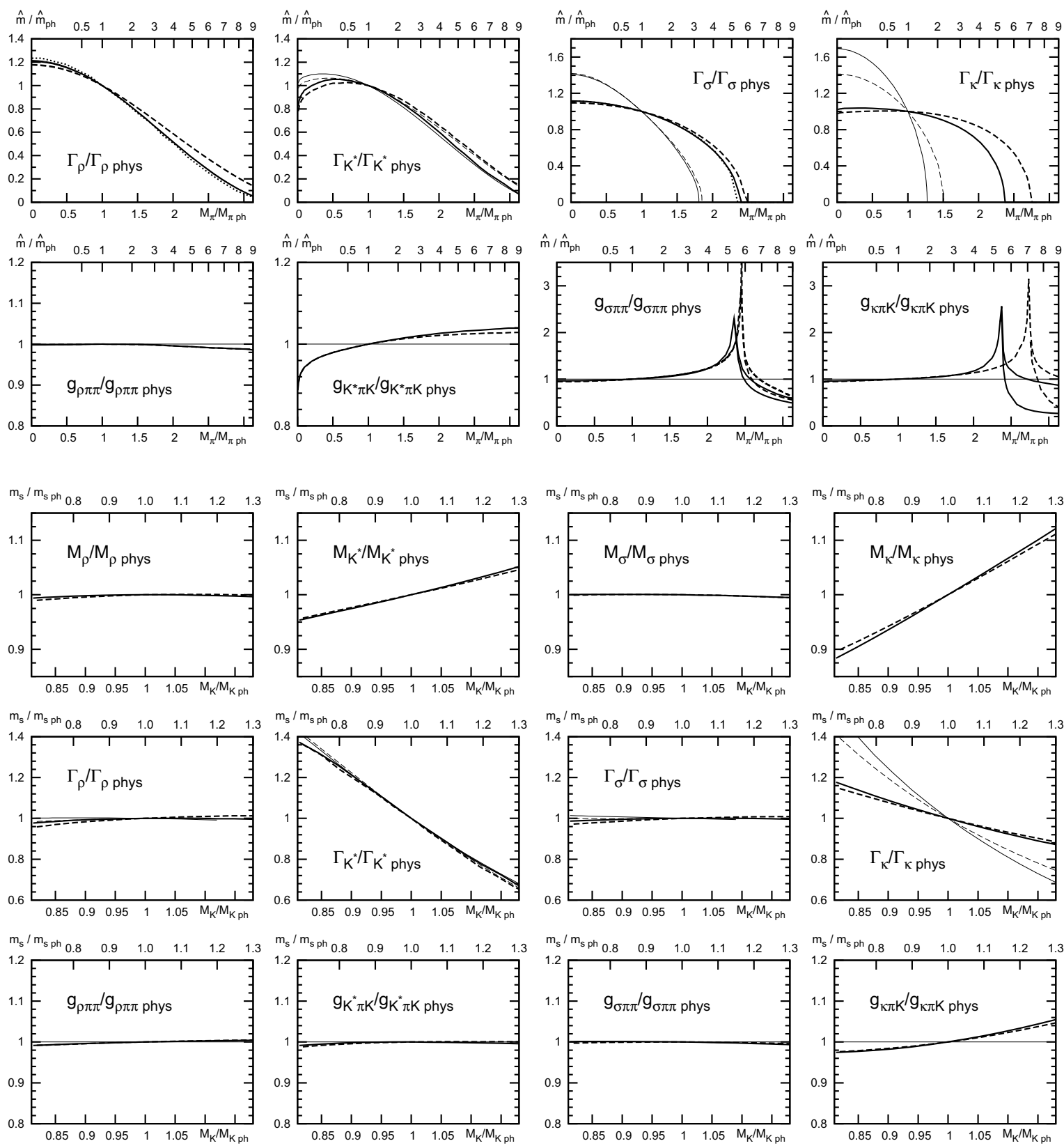

FIGURE 3. Dependence of the $\rho(770), K^{*}(892), f_{0}(600)$ and $\kappa(800)$ mass, width and coupling to two mesons with respect to $\hat{m}$ (horizontal upper scale) or $M_{\pi}$ (horizontal lower scale) for the three upper rows and with respect to $m_{s}$ (horizontal upper scale) or $M_{K}$ (horizontal lower scale) for the three lower rows. Note that all quantities are normalized to their physical values. The thick continuous and dashed lines correspond to Fit I and Fit II in Ref. [3], respectively. The $\rho(770)$ and $f_{0}(600)$ dependence on $\hat{m}$ is very compatible with that in [2] (dotted lines). Continuous (dashed) thin lines show the dependence of the widths from the change of phase space only, assuming a constant coupling of the resonances to two mesons, using Fit I (II). 
Copyright of AIP Conference Proceedings is the property of American Institute of Physics and its content may not be copied or emailed to multiple sites or posted to a listserv without the copyright holder's express written permission. However, users may print, download, or email articles for individual use. 